\title{
Comparison of two kinds of nanomedicine for targeted gene therapy: premodified or postmodified gene delivery systems
}

This article was published in the following Dove Press journal:

International Journal of Nanomedicine

16 April 2012

Number of times this article has been viewed

\author{
Zhaoshun Jiang \\ Cong Sun \\ Zhaohui Yin \\ Fang Zhou \\ Linfu Ge \\ Ximin Liu \\ Fansheng Kong \\ General Hospital of Ji'nan Command, \\ PLA, Ji'nan, Shandong, China
}

Correspondence: Fansheng Kong Department of Hematology, General Hospital of Ji'nan Command, PLA, 25 Shifan Road, Ji'nan 25003I,

People's Republic of China

Tel +86053 I 5I665200

Email kongfanshengphd@yahoo.com.cn
Background: The applications of ligand-polyethylene glycol (PEG)-modified nanocarriers have now emerged, as well as recognized strategies to provide the vectors with active targeting properties. In this research, premodification and postmodification were compared using the same ligand, ie, a novel conjugated mannan-containing PEG and L- $\alpha$-phosphatidylethanolamine (PE).

Methods: Premodified and postmodified solid lipid nanoparticles were prepared and the characteristics of the two kinds of vehicles were evaluated. The modified vectors were then administered intravenously to rats and the in vivo targeting behavior of the complexes was investigated in liver macrophages.

Results: By carefully formulating the carriers with an optimal ratio of mannan-containing PEG-PE, postmodified vehicles displayed more efficient gene expression in rat Kupffer cells both in vitro and in vivo.

Conclusion: Postmodified gene carriers are superior to premodified gene vectors, although the latter is also promising for targeted gene delivery. This discovery could guide our future research.

Keywords: gene therapy, nanomedicine, mannan, polyethylene glycol, L- $\alpha$ phosphatidylethanolamine, ligands, premodification, postmodification

\section{Introduction}

The prospect of gene nanomedicine holds great promise for alleviation and cure of many presently untreatable diseases. ${ }^{1}$ Ideally, a gene delivery system should be stable, biocompatible, nontoxic, cost-effective, and able to be delivered (targeted) to a specific site, transfer highly anionic exogenous genetic materials, and have maximum therapeutic efficacy. ${ }^{2}$ However, the lack of efficient site-specific delivery systems has impeded the realization of gene therapy in practice. ${ }^{3}$

Compared with viral vectors, nonviral pharmaceutical vectors, such as polymeric nanoparticles, liposomes, micelles, nanocapsules, solid lipid nanoparticles (SLN), niosomes, and other vectors are potentially less toxic, less immunogenic, relatively easy to produce on a large scale, and able to be modified. ${ }^{46}$ Surface modification of nanovectors could improve their longevity, bioavailability, targeting ability, and intracellular penetration, and thus provide a range of opportunities for efficient delivery of anticancer drugs, genes, and diagnostic agents..$^{7-10}$

In terms of modification, they could be classified in two different ways, ie, premodification and postmodification. Premodification complexes could be prepared by mixing drugs/genes with modified vectors, while postmodification complexes can be obtained by surface modification using conventional drug-/gene-loaded carriers. ${ }^{7}$ 
Examples include the post-pegylated lipoplexes reported by Peeters et al to be more promising vehicles for gene delivery than pre-pegylated lipoplexes in retinal pigment epithelium cells. ${ }^{11}$ Both premodification and postmodification methods have advantages. Premodified vehicles could be more stable, and loading of drugs/genes would not change the targeting ability of the vectors, ${ }^{12-15}$ whereas postmodified carriers might be modified more efficiently and have better targeting capability. ${ }^{16,17}$ However, which method is better for targeted gene delivery is unknown and is a focus of our ongoing investigations.

Targeted macrophages are one of the promising strategies for treating diseases such as human immunodeficiency virus infection, because macrophages play a major role in the immune response to foreign antigens. ${ }^{18}$ Kupffer cells are liver-specific resident macrophages that play an integral part in the physiological homeostasis of the liver. They have a significant role in the acute and chronic responses of the liver to bacterial and viral infections, toxic or carcinogenic attack, and mediate hepatotoxicity. ${ }^{19}$ It has been shown that human and murine macrophages express the mannose receptor on their surface, and several studies have confirmed the feasibility of using mannose or mannan-modified nanocarriers to target macrophages. ${ }^{20}$

In the present study, a novel conjugated ligand, mannanpolyethylene glycol- L- $\alpha$-phosphatidylethanolamine (MNPEG-PE) was synthesized, and MN-PEG-PE-modified SLN were investigated. In this study we compared the two strategies for modification using the same ligands (MN-PEG-PE) in an attempt to identify which kind of nanomedicine is preferable for targeted gene therapy, ie, premodified or postmodified gene-loaded SLN/DNA. Cationic SLN were used as the vectors and plasmid-enhanced green fluorescent protein (pEGFP) was used as the model gene. The properties of these two kinds of modified vehicles were compared using in vitro and in vivo transfection in a rat Kupffer cell model. Unmodified gene-loaded complexes were used as controls.

\section{Materials and methods Materials}

Stearic acid, dimethyldioctadecylammonium bromide, mannan, PE, concanavalin A (Con A), and MTT (3-[4,5dimehyl-2-thiazolyl]-2,5-diphenyl-2H-tetrazolium bromide) were purchased from Sigma-Aldrich Co, Ltd (St Louis, MO). Injectable soya lecithin was obtained from Shanghai Taiwei Pharmaceutical Co, Ltd (Shanghai, China). Butyl carbonyl (Boc)-NH-PEG ${ }_{2000}-\mathrm{COOH}$ was purchased from Shanghai Yarebio Co, Ltd (Shanghai, China). pEGFP-N1 was provided by Shandong University (Shandong, China). Quant-iT ${ }^{\text {тM }}$ PicoGreen ${ }^{\circledR}$ dsDNA quantitation reagent was obtained from Invitrogen by Life Technologies (Carlsbad, CA). All other chemicals were of analytical grade or higher.

\section{Animals}

Adult male Wistar rats (aged 10-12 weeks) were purchased from the Medical Animal Test Center of Shandong Province and housed under standard laboratory conditions. All animal experiments complied with the requirements of the National Act on the Use of Experimental Animals (People's Republic of China).

\section{Synthesis of MN-PEG-PE ligands}

MN-PEG-PE ligands were synthesized as described in Figure 1. Boc-NH-PEG-COOH $100 \mathrm{mg}$ was dissolved in dimethyl sulfoxide (DMSO) and stirred with PE $36 \mathrm{mg}$ as a mixture. 1-[3(dimethylamino)propyl]-3-ethylcarbodiimide (EDC $\cdot \mathrm{HCl})$ $72 \mathrm{mg}$ and triethylamine (1 equivalent of $\mathrm{EDC} \cdot \mathrm{HCl})$
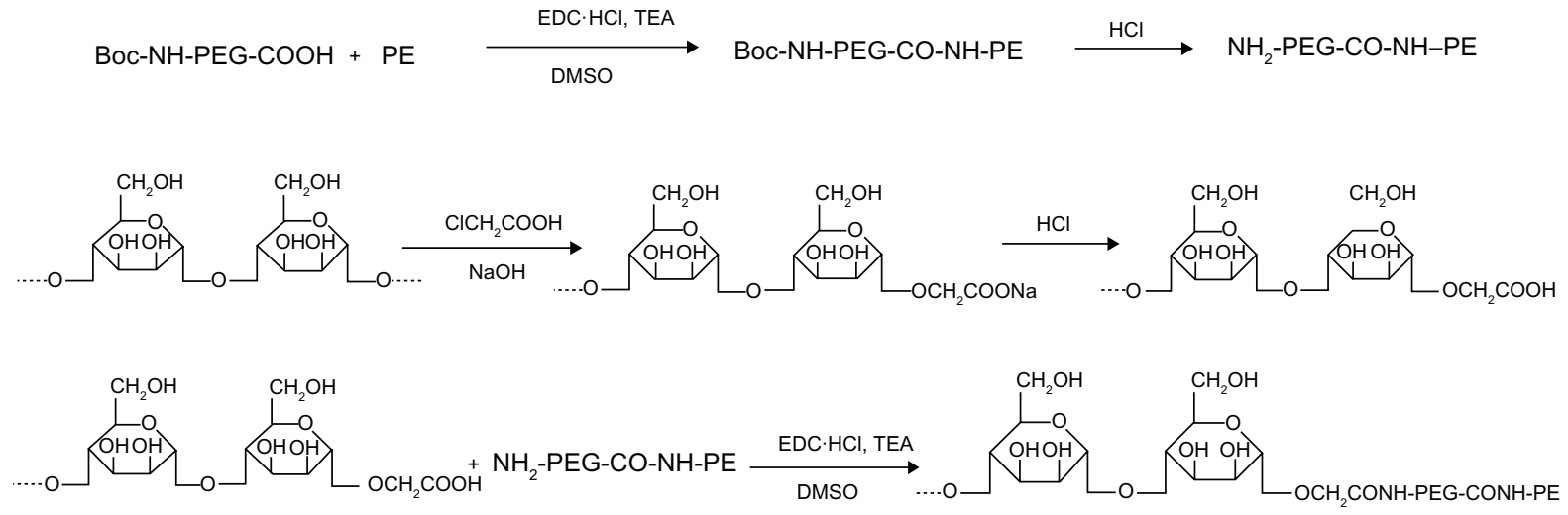

Figure I General reaction scheme for synthesis of MN-PEG-PE.

Abbreviations: DMSO, dimethylsulfoxide; MN, mannan; PEG, polyethylene glycol; PE, L- $\alpha$-phosphatidylethanolamine; TEA, triethylamine. 
were dissolved in DMSO and added dropwise into the mixture in an ice bath and stirred for 36 hours, following which concentrated hydrochloric acid was used to detach the Boc group, to produce $\mathrm{NH}_{2}$-PEG-CO-NH-PE. Mannan $100 \mathrm{mg}$ was dissolved in $1 \mathrm{M}$ sodium hydroxide $1 \mathrm{~mL}$ and stirred for 30 minutes for alkalinization. Chloroacetic acid $(20 \%, 1 \mathrm{~mL})$ was then added to the solution and stirred in an oil bath at $60^{\circ} \mathrm{C}$ for 6 hours. After that, $1 \mathrm{M}$ hydrochloric acid was added until a $\mathrm{pH}$ of 2-3 was reached, to complete the carboxymethylation of mannan. Carboxymethylated mannan was then stirred with $\mathrm{NH}_{2}$-PEG-CO-NH-PE in DMSO solution, and $\mathrm{EDC} \cdot \mathrm{HCl}$ mixed with triethylamine (1 equivalent of $\mathrm{EDC} \cdot \mathrm{HCl}$, in DMSO) was added dropwise into the solution in an ice bath and stirred for 24 hours. DMSO was moved by rotary evaporation, and the product was dialyzed against Milli-Q water (Millipore Corporation, Billerica, MA) for 24 hours for final formation of MN-PEG-PE.

\section{Preparation of cationic SLN}

SLN were prepared using the nanoprecipitation method (solvent displacement technique). ${ }^{20-22}$ Stearic acid $50 \mathrm{mg}$ and injectable soya lecithin $30 \mathrm{mg}$ were accurately weighed and dissolved in $10 \mathrm{~mL}$ of acetone. The organic phase was added dropwise into the $0.2 \%$ dimethyldioctadecylammonium bromide solution, under stirring at $600 \mathrm{rpm}$ and at room temperature. When complete evaporation of the organic solvent had occurred, the redundant stabilizers were separated by ultracentrifugation at $1000 \mathrm{~g}$ and at $4{ }^{\circ} \mathrm{C}$ for 20 minutes. The pellet was vortexed and resuspended in Milli-Q water, washed three times, filtered through a $0.45 \mu \mathrm{m}$ membrane, and adjusted to $\mathrm{pH} 7.0 \pm 0.2$ with sodium hydroxide. The SLN suspensions obtained were stored at $2^{\circ} \mathrm{C}-8^{\circ} \mathrm{C}$.

\section{Preparation and optimization of MN-PEG-PE-modified SLN/DNA}

\section{Premodification}

Pre-MN-PEG-PE-modified SLN/DNA (A-MN-SLN/DNA) complexes were prepared by firstly modifying the SLN with MN-PEG-PE to form new vehicles, followed by complexation of the vectors with pEGFP (Figure 2A).

Briefly, MN-PEG-PE ligands were dissolved in $5 \mathrm{~mL}$ of phosphate-buffered saline ( $\mathrm{pH} 7.4)$. The solution was then added dropwise into $20 \mathrm{~mL}$ of SLN and stirred at $600 \mathrm{rpm}$ and room temperature, leading to immediate modification. Subsequently, free MN-PEG-PE was removed from the modified SLN/DNA by gel chromatography using a Sephadex ${ }^{\circledR}$ G-50 column. The complexes obtained were resuspended in Milli-Q water, washed three times, and filtered through a membrane with an $0.80 \mu \mathrm{m}$ pore size to obtain MN-PEG-PE modified SLN.

MN-PEG-PE ligands were continuously coated onto the surface of the SLN. During the progress of modification, the positive surface charge would have been masked, and this process caused a decrease in the zeta potential. The optimum formulation was obtained when there was no obvious change in the potential. To optimize the modification ratio, MN-PEG-PE ligands dissolved in phosphate-buffered saline were designed at different weight ratios to the SLN (w/w). The change in zeta potential of the complexes was determined using a Zetasizer Nano-ZS instrument (Malvern, Worcestershire, UK).

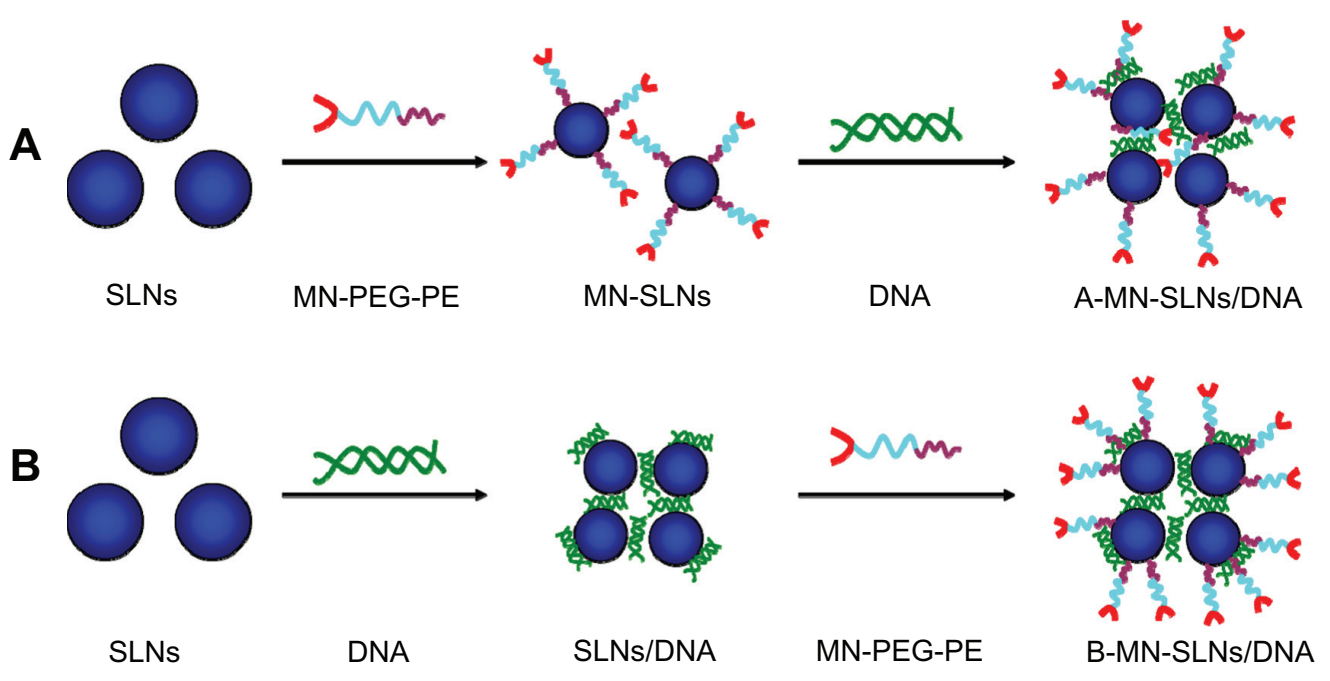

Figure 2 Preparation of pre- (A) and post- (B) MN-PEG-PE-modified SLN/DNA.

Abbreviations: SLN, solid lipid nanoparticles; MN, mannan; PEG, polyethylene glycol; PE, L- $\alpha$-phosphatidylethanolamine; A-MN-SLNs/DNA, pre-MN-PEG-PE-modified SLN/DNA; B-MN-SLNs/DNA, post-MN-PEG-PE-modified SLN/DNA. 
The reporter gene, pEGFP, was mixed with the premodified SLN by vortexing the suspension with a $1 \mathrm{mg} / \mathrm{mL}$ solution of pEGFP for 20 seconds. Incubation of the mixture for 30 minutes at room temperature facilitated formation of the A-MN-SLN/DNA.

\section{Postmodification}

Post-MN-PEG-PE-modified SLN/DNA (B-MN-SLN/DNA) complexes were prepared by incubating the SLN with pEGFP to form SLN/DNA, followed by modification of SLN/DNA with MN-PEG-PE (Figure 2B). Briefly, pEGFP was mixed with SLN by vortexing the particles with a $1 \mathrm{mg} / \mathrm{mL}$ solution of pEGFP for 20 seconds. Incubation of the mixture for 30 minutes at room temperature facilitated formation of SLN/DNA.

MN-PEG-PE ligands were dissolved in $5 \mathrm{~mL}$ of phosphate-buffered saline ( $\mathrm{pH}$ 7.4). The solution was then added dropwise to $20 \mathrm{~mL}$ of SLN/DNA complexes and stirred at $600 \mathrm{rpm}$ in room temperature, leading to immediate modification. Subsequently, free MN-PEG-PE was removed from the modified SLN/DNA by gel chromatography using a Sephadex G-50 column. The complexes obtained were resuspended in Milli-Q water, washed three times, and filtered through a membrane with a pore size of $0.80 \mu \mathrm{m}$ to obtain B-MN-SLN/DNA. During this procedure, the MNPEG-PE ligands were continuously coated onto the surface of the SLN/DNA which would also have caused a decrease in the zeta potential. To optimize the modification ratio, MN-PEG-PE ligands dissolved in phosphate-buffered saline were designed at different weight ratios to the SLN (w/w), and the zeta potential of the complexes was determined. PEG-PE ligands without mannan were dissolved in $5 \mathrm{~mL}$ of phosphate-buffered saline and modified onto the SLN/ DNA surface using the same method as described earlier for preparation of PEG-PE-SLN/DNA as a control.

\section{Characterization of premodified and postmodified SLN/DNA \\ Gene-loading capacities: PicoGreen fluorometry assay}

A PicoGreen fluorometry assay was used to quantitate and compare the gene-loading abilities of premodified and postmodified SLN/DNA. ${ }^{23-25}$ The pEGFP was isolated from the A-MN-SLN/DNA and B-MN-SLN/DNA by centrifugation at $1000 \mathrm{~g}$ and $4^{\circ} \mathrm{C}$ for 20 minutes. The concentration of pEGFP was determined by fluorescence, and compared with the supernatant from SLN. The amount of pEGFP loaded into the SLN was calculated according to the linear calibration curve for pEGFP (10 to $2000 \mathrm{ng} / \mathrm{mL}, \mathrm{R}^{2}=0.9993$ ). Gene loading quantity $(\%)=($ total amount of pEGFP - amount of free $\mathrm{pEGFP}$ )/total amount of DNA $\times 100$.

\section{Determination of modification: Con A agglutination study}

Binding of the terminal $\alpha$-mannose residues to Con A causes agglutination of the complexes in solution, resulting in an increase in turbidity. ${ }^{26-28}$ To confirm that the MN-PEG-PE ligands were successfully modified onto the SLN/DNA surface, a Con A agglutination assay was performed. Briefly, $100 \mu \mathrm{L}$ each of A-MN-SLN/DNA, B-MN-SLN/DNA, and unmodified SLN/DNA complexes were added separately into $500 \mu \mathrm{L}$ of Con $\mathrm{A}(1 \mathrm{mg} / \mathrm{mL})$ in phosphate-buffered saline ( $\mathrm{pH}$ 7.4) with $5 \mathrm{mM}$ of $\mathrm{CaCl}_{2}$ and $5 \mathrm{mM}$ of $\mathrm{MgCl}_{2}$. The increase in turbidity at $360 \mathrm{~nm}$ (OD360) was monitored for 180 seconds.

\section{Isolation and culture of Kupffer cells}

Kupffer cells were isolated from Wistar rats under pentobarbitone anesthesia using a method described elsewhere. ${ }^{29}$ The rat portal veins were cannulated and perfused with Hank's buffered salt solution for 10 minutes at room temperature. During this time, the liver was excised and the perfusate discarded. The liver was then perfused with $0.2 \%$ pronase (60 $\mathrm{mL}$ at room temperature) which was discarded. Next, the liver was perfused with a recirculating solution of $0.05 \%$ pronase and $0.05 \%$ collagenase $(60 \mathrm{~mL}$ at room temperature) until the liver was digested, as judged by softening of the liver parenchyma beneath the capsule. The liver was then cut into small pieces, suspended in $100 \mathrm{~mL}$ of solution containing $0.02 \%$ pronase, $0.05 \%$ collagenase, and $0.005 \%$ DNase, and agitated at room temperature for 20 minutes. Following digestion, the liver homogenate was filtered through sterile gauze and centrifuged (at $1000 \mathrm{~g}$ and $4^{\circ} \mathrm{C}$ for 10 minutes). The supernatant was removed and the pellet was resuspended in $10 \mathrm{~mL}$ of Percoll gradient. Aliquots $(5 \mathrm{~mL})$ of this cell suspension were added to $5 \mathrm{~mL}$ aliquots of Percoll gradient. These were carefully overlaid with $5 \mathrm{~mL}$ of Hank's buffered salt solution and centrifuged at $1000 \mathrm{~g}$ and $4^{\circ} \mathrm{C}$ for 20 minutes. The nonparenchymal cell-enriched layer observed at the interface between the two layers was carefully harvested and diluted with $10 \mathrm{~mL}$ of Hank's buffered salt solution. The suspension was then centrifuged (1000 g and $4^{\circ} \mathrm{C}$ for 20 minutes) to precipitate the Kupffer cells, which were then seeded into a 96-well microtiter plate at a density of $2 \times 10^{4}$ cells/well in $200 \mu \mathrm{L}$ RPMI-1640 supplemented with $10 \%$ fetal bovine serum and antibiotics. After incubation 
at $37^{\circ} \mathrm{C}$ for 2 hours in a $5 \% \mathrm{CO}_{2}$ atmosphere, the culture medium was replaced by $200 \mu \mathrm{L}$ of fresh RPMI-1640 to yield the purified Kupffer cells.

The isolated rat liver Kupffer cells were defined, and about $8.15 \times 10^{7}$ cells were obtained, with a purity of about $91.2 \%\left(8.94 \times 10^{7} /\right.$ rat liver nonparenchymal cells $)$. The isolated and purified rat Kupffer cells retained their in vivo morphological, biological, and immunological characteristics.

\section{In vitro cytotoxicity evaluation}

The in vitro cytotoxicity of A-MN-SLN/DNA and B-MNSLN/DNA against Kupffer cells was evaluated by MTT assay. ${ }^{30}$ The Kupffer cells were incubated with modified and unmodified SLN/DNA at various concentrations (10, 20, 50, 100 , and $200 \mu \mathrm{g} / \mathrm{mL}$ ) for 48 hours at $37^{\circ} \mathrm{C}$ and in a $5 \% \mathrm{CO}_{2}$ atmosphere. Cell viability was then assessed by MTT assay. Next, MTT $5 \mathrm{mg} / \mathrm{mL}$ in phosphate-buffered saline was added to each well and the plate was incubated for a further 4 hours at $37^{\circ} \mathrm{C}$ in a $5 \% \mathrm{CO}_{2}$ atmosphere. The MTT containing the medium was then removed, and the crystals formed by living cells were dissolved in $100 \mu \mathrm{L}$ of DMSO. The absorbance at $570 \mathrm{~nm}$ was determined by a microplate reader. Untreated cells were taken as a control with $100 \%$ viability, and cells without the addition of MTT were used as a blank to calibrate the spectrophotometer to zero absorbance. The relative cell viability (\%) compared with control cells was calculated using (Abssample/Abscontrol) $\times 100$.

\section{In vitro release of MN-SLN/DNA}

The in vitro release studies of premodified and postmodified MN-SLN/DNA were performed in phosphate-buffered saline (pH 7.4). ${ }^{31}$ Typically, aliquots of complexes were suspended in $1 \mathrm{~mL}$ of phosphate-buffered saline (in Eppendorf ${ }^{\mathbb{R}}$ tubes) and vortexed for 30 seconds. The tubes were then placed in a $37^{\circ} \mathrm{C}$ shaking water bath at $100 \mathrm{rpm}$. Separate tubes were used for different data points. At predetermined time intervals, the suspensions were centrifuged at $1500 \mathrm{~g}$ for 30 minutes, and the amount of DNA released in the supernatant was analyzed using the PicoGreen assay. Background readings were obtained using the supernatants from the blank SLN.

\section{In vitro transfection analysis}

The Kupffer cells were seeded into 24-well plates at a density of $8 \times 10^{4}$ cells/well in $1 \mathrm{~mL}$ of RPMI-1640 with $10 \%$ fetal bovine serum. After about 24 hours, the medium was replaced by $500 \mu \mathrm{L}$ of transfection medium containing
A-MN-SLN/DNA and B-MN-SLN/DNA. Unmodified SLN/DNA complexes and PEG-PE ligand-modified SLN/ DNA without mannan (PEG-PE-SLN/DNA) were used as controls. The original incubation medium was replaced with $1 \mathrm{~mL}$ of complete medium after incubation at $37^{\circ} \mathrm{C}$ for 4 hours in a $5 \% \mathrm{CO}_{2}$ atmosphere. The cells were incubated and studied until 72 hours after transfection. The fluorescent cells were observed using an inversion fluorescence microscope, at which time pictures were taken for the record.

\section{In vivo gene delivery}

Adult male Wistar rats were divided into five groups (six rats in each group) and injected intravenously with $1 \mathrm{~mL}$ of naked DNA, SLN/DNA, PEG-PE-SLN/DNA, A-MN-SLN/ DNA, or B-MN-SLN/DNA (containing $60 \mu \mathrm{g}$ of pEGFP). At predetermined time intervals $(24,48$, and 72 hours), the rats were euthanized and their Kupffer cells were isolated and cultured as described earlier. The fluorescent cells were observed using an inversion fluorescence microscope, at which time pictures were taken for the record. After that, the cells were washed with $1 \mathrm{~mL}$ of phosphate-buffered saline (100 g and $4^{\circ} \mathrm{C}$ for 5 minutes) and were detached with trypsin/ EDTA. The supernatant was discarded and resuspended with $300 \mu \mathrm{L}$ of phosphate-buffered saline and added into the flow cytometer to quantitate the amount of Kupffer cells which has been successfully transfected.

\section{Statistical analysis}

All studies were repeated three times and the measurements were carried out in triplicate. Results were reported as the mean \pm standard deviation. Statistical significance was analyzed using the Student's $t$-test. Differences between experimental groups were considered significant at $P<0.05$.

\section{Results \\ Structure confirmation of MN-PEG-PE ligands}

The structure of MN-PEG-PE was confirmed by infrared spectroscopy and nuclear magnetic resonance ( ${ }^{1} \mathrm{H}$ NMR) spectroscopy. Infrared $v / \mathrm{cm}^{-1}: 3517.3(-\mathrm{NH}-,-\mathrm{OH}) ; 1820.4$ $(-\mathrm{C}=\mathrm{O}) ; 1667.9(-\mathrm{HN}-\mathrm{CO}-) ; 1635.2(-\mathrm{HN}-\mathrm{CO}-$, evidence of the mannan linked with PEG-PE); and 1093.2 ( $-\mathrm{C}-\mathrm{O}-\mathrm{C}-$, secondary alcohol structure in mannan). ${ }^{1} \mathrm{H}$ NMR (DMSO-d6, $300 \mathrm{MHz}) \delta: 2.47\left(\mathrm{CH}_{2} \mathrm{CO}\right), 3.26\left(\mathrm{CH}_{2} \mathrm{~N}\right) 6.05(\mathrm{NH})$, and $\delta(3.0-6.5)$. Similar peaks were observed as the spectra of mannan, ie, $\delta(0-3.0)$, and the peaks were in accordance with PEG-PE. The production rate was around $70 \%$. 


\section{Optimization of modification ratio} and quantitation of binding capacity

The optimization of ligand to carrier ratio and binding capacity of the optimized vectors were as follows:

\section{Premodified SLN/DNA}

MN-PEG-PE ligands were previously coated onto the SLN surface. The ratio of ligands to carriers was optimized by measuring the change in zeta potential. As illustrated in Figure 3A, the optimized ratio of MN-PEG-PE to SLN was 40\%. This ratio was used for further experiments. MN-SLN were then incubated with pEGFP to form A-MN-SLN/DNA. The gene loading efficiency of A-MN-SLN/DNA was around 80\%.

\section{Postmodified SLN/DNA}

SLN were firstly incubated with pEGFP to form SLN/DNA. The MN-PEG-PE ligands were next coated onto the SLN/DNA surface to form B-MN-SLN/DNA. As shown in Figure 3B, the optimized ratio of MN-PEG-PE to SLN/DNA was 40\%. This ratio was used for the further experiments. The gene loading efficiency of B-MN-SLN/DNA was around $87 \%$.

\section{Characterization of premodified and postmodified MN-SLN/DNA}

The mean particle size, polydispersity index, and zeta potential of SLN, MN-SLN, SLN/DNA, A-MN-SLN/DNA,

A

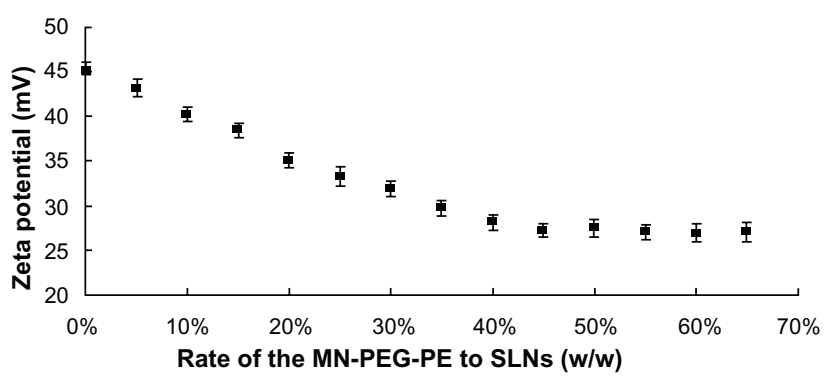

B

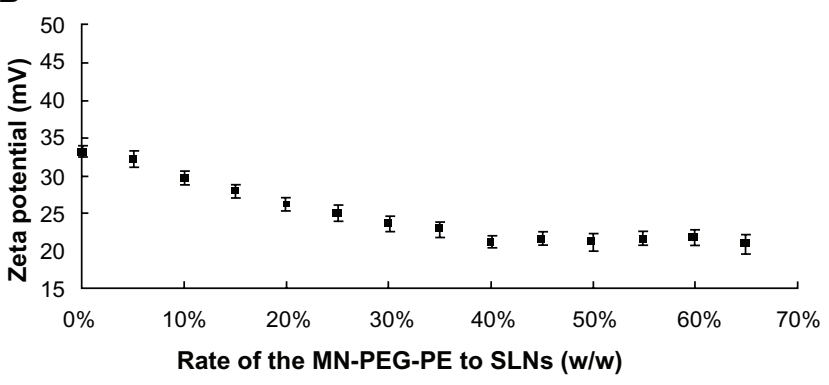

Figure 3 Optimization of the modification ratios for (A) pre-MN-PEG-PE-modified SLN/DNA and (B) post-MN-PEG-PE-modified SLN/DNA.

Abbreviations: SLN, solid lipid nanoparticles; MN, mannan; PEG, polyethylene glycol; PE, L- $\alpha$-phosphatidylethanolamine. and B-MN-SLN/DNA were characterized and are shown in Table 1 .

\section{Determination of MN-PEG-PE modification}

A Con A agglutination assay was used to confirm further that the MN-PEG-PE ligands were successfully modified onto the vector surface. As shown in Figure 4, the turbidity of A-MNSLN/DNA and B-MN-SLN/DNA appeared to increase, while the SLN and SLN/DNA formulations appeared to show no significant increase in turbidity.

\section{In vitro cytotoxicity evaluation}

The in vitro cytotoxicity of A-MN-SLN/DNA and B-MNSLN/DNA against Kupffer cells was evaluated at various concentrations by MTT assay. The cell viability of the vectors over the studied concentration range $(10-200 \mu \mathrm{g} / \mathrm{mL})$ was between $80 \%$ and $100 \%$ compared with controls (Figure 5).

\section{In vitro release and in vitro}

\section{transfection analysis}

The in vitro release profiles for A-MN-SLN/DNA and B-MNSLN/DNA are shown in Figure 6. Both the premodified and postmodified SLN/DNA reached over $80 \%$ gene release after 72 hours. The in vitro transfection efficiency of A-MN-SLN/ DNA and B-MN-SLN/DNA in Kupffer cells after 72 hours of transfection was analyzed and compared with naked DNA, unmodified SLN/DNA, and PEG-PE-SLN/DNA (Figure 7). B-MN-SLN/DNA showed higher transfection efficiency than A-MN-SLN/DNA at 48 and 72 hours after transfection $(P<0.05)$.

\section{In vivo gene delivery}

The in vivo transfection efficiency of A-MN-SLN/DNA and B-MN-SLN/DNA was observed and is shown in Figure 8.

Table I Particle size and zeta potential of different vectors

\begin{tabular}{lcll}
\hline $\begin{array}{l}\text { Characteristics/ } \\
\text { samples }\end{array}$ & $\begin{array}{l}\text { Mean particle } \\
\text { size }(\mathbf{n m})\end{array}$ & $\begin{array}{l}\text { Polydispersity } \\
\text { index }\end{array}$ & $\begin{array}{l}\text { Zeta potential } \\
(\mathbf{m V})\end{array}$ \\
\hline SLN & $87.5 \pm 5.2$ & $0.11 \pm 0.02$ & $+45.2 \pm 1.5$ \\
MN-SLN & $153.1 \pm 4.3$ & $0.20 \pm 0.02$ & $+28.5 \pm 2.3$ \\
SLN/DNA & $121.8 \pm 5.8$ & $0.15 \pm 0.03$ & $+33.1 \pm 2.4$ \\
Pre-MN-PEG- & $223.6 \pm 5.3$ & $0.22 \pm 0.05$ & $+20.4 \pm 2.1$ \\
PE-modified & & & \\
SLN/DNA & & & \\
Post-MN-PEG- & $185.2 \pm 4.1$ & $0.11 \pm 0.04$ & $+21.3 \pm 1.7$ \\
PE-modified & & & \\
SLN/DNA & & & \\
\hline Abbrevition & & &
\end{tabular}

Abbreviations: SLN, solid lipid nanoparticles; MN, mannan; PEG, polyethylene glycol; PE, L- $\alpha$-phosphatidylethanolamine. 


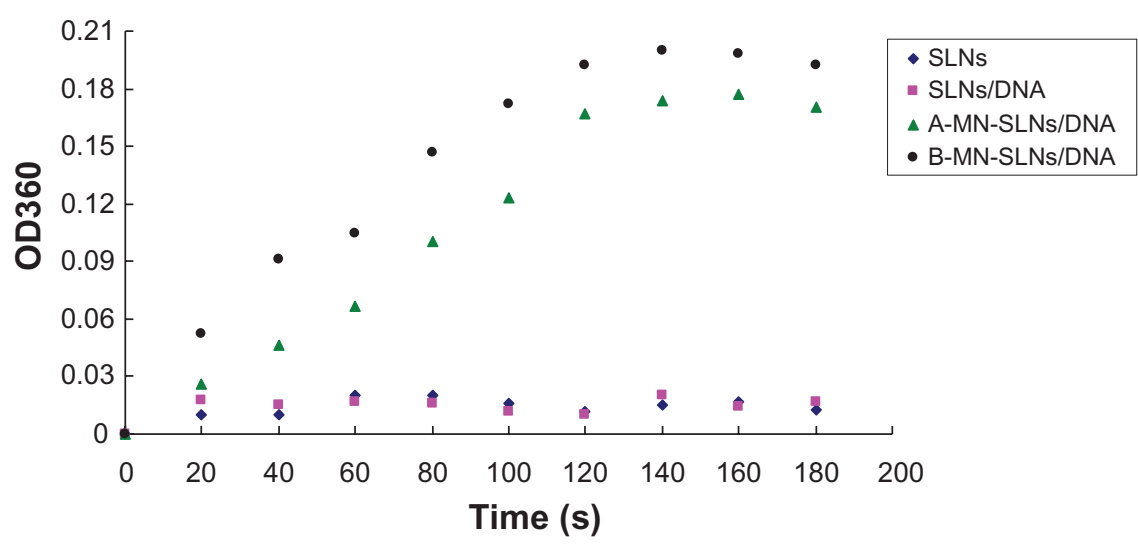

Figure 4 Con A agglutination assay of different vectors.

Abbreviations: SLN, solid lipid nanoparticles; A-MN-SLNs/DNA, pre-MN-PEG-PE-modified SLN/DNA; B-MN-SLNs/DNA, post-MN-PEG-PE-modified SLN/DNA; MN, mannan; PEG, polyethylene glycol; PE, L- $\alpha$-phosphatidylethanolamine.

B-MN-SLN/DNA had higher transfection efficiency at different time intervals compared with the other vectors. Flow cytometry was used to quantitate further the amount of cells which had been successfully transfected. As shown in Figure 9, B-MN-SLN/DNA showed a remarkably higher transfection efficiency compared with A-MN-SLN/DNA and unmodified SLN/DNA $(P<0.05)$.

\section{Discussion}

The applications of ligand-PEG modification of nanocarriers have emerged as well recognized strategies to provide these vectors with active targeting properties using a wide assortment of covalently attached targeting ligands. ${ }^{32} \mathrm{~A}$ series of PEG-PE conjugates has been used for the modification of various vehicles to create active targeting nanocarriers capable of spontaneous accumulation at specific sites. ${ }^{33-36}$
In this research, novel conjugated mannan-containing PEG-PE ligands were synthesized and MN-PEG-PEmodified SLN were investigated. Here, mannan is the target moiety which can bind to the mannose receptor in the macrophage, and PEG-PE is the spacer linked onto the surface of the SLN. It has been suggested that the length and flexibility of the spacer between the carbohydrate head group and carrier surface mainly influences the target specificity and uptake of vectors by macrophages. ${ }^{37}$ Use of PEG-PE conjugates has been reported and TATp-PEG ${ }_{2000}$-PE conjugates have been applied for modification of nanocarriers to form active targeting vectors, ${ }^{38}$ so $\mathrm{PEG}_{2000}$-PE was used as the anchor.

In our research, we are investigating which form of modification is better, ie, premodification to form newly modified vectors or postmodification after DNA has been loaded. In this study, we compared the two methods of

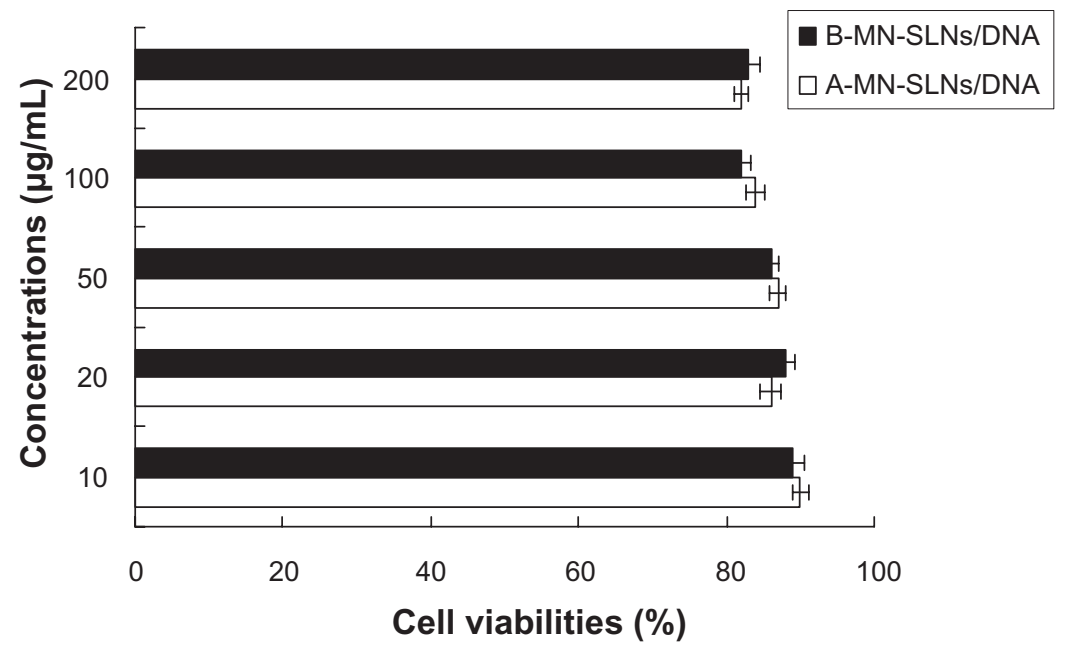

Figure 5 Viability of Kupffer cells targeted with A-MN-SLN/DNA and B-MN-SLN/DNA.

Abbreviations: SLN, solid lipid nanoparticles; A-MN-SLNs/DNA, pre-MN-PEG-PE-modified SLN/DNA; B-MN-SLNs/DNA, post-MN-PEG-PE-modified SLN/DNA; $\mathrm{MN}$, mannan; PEG, polyethylene glycol; PE, L- $\alpha$-phosphatidylethanolamine. 


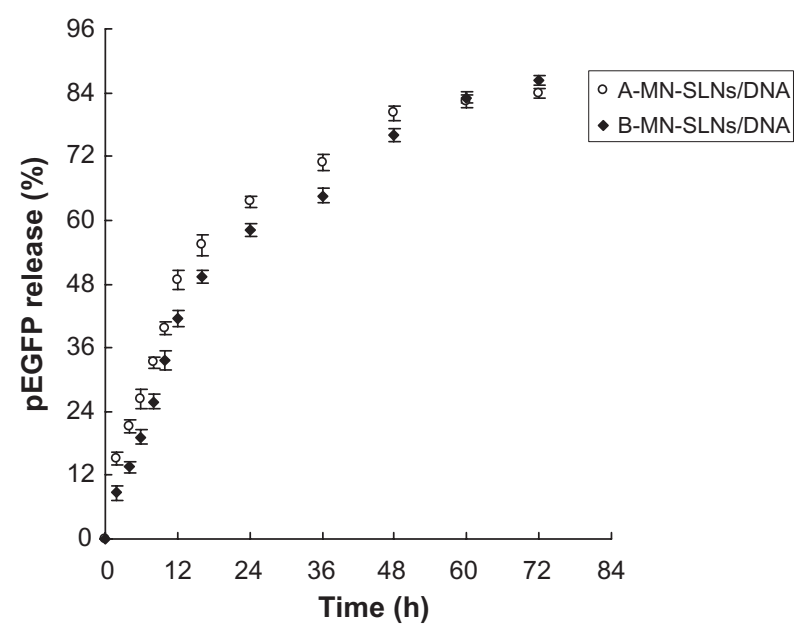

Figure 6 In vitro release profiles of A-MN-SLN/DNA and B-MN-SLN/DNA. Abbreviations: SLN, solid lipid nanoparticles; A-MN-SLNs/DNA, pre-MN-PEGPE-modified SLN/DNA; B-MN-SLNs/DNA, post-MN-PEG-PE-modified SLN/DNA; MN, mannan; PEG, polyethylene glycol; PE, L- $\alpha$-phosphatidylethanolamine; pEGFP, plasmid-enhanced green fluorescent protein.

modification using the same ligands. Our investigation began with preparation and optimization of the MN-PEG-PE modification ratio for the two kinds of modification. As shown in Figure 3, A-MN-SLN/DNA refers to SLN which had been previously modified by MN-PEG-PE to form MN-SLN and then incubated with pEGFP; whereas B-MN-SLN/DNA were prepared by firstly binding DNA with SLN followed by coating of the MN-PEG-PE ligands. The modification ratios of A-MN-SLN/DNA and B-MN-SLN/DNA were approximately the same $(40 \%$, ligands/vectors, w/w). The gene loading efficiency of B-MN-SLN/DNA was slightly higher than that of A-MN-SLN/DNA (80\%-87\%). This may be explained as follows. The complexation of genes by SLN was void-free, and subsequent coating of ligands did not affect the gene binding capacity, whereas premodified SLN were coated with ligands first and this may have had some influence on gene complexation caused a slight decrease in loading efficacy.

When the modification ratios of the two kinds of vectors had been determined, A-MN-SLN/DNA and B-MN-SLN/ DNA were prepared and characterized. The mean particle sizes of A-MN-SLN/DNA and B-MN-SLN/DNA were about $223 \mathrm{~nm}$ and $185 \mathrm{~nm}$, respectively. The premodified vectors was larger than the postmodified vectors, which might be because complexation of A-MN-SLN with DNA was not as tight as for B-MN-SLN/DNA. The polydispersity index of B-MN-SLN/DNA was smaller than for A-MN-SLN/DNA, which had a relatively narrow size distribution. These differences in physical properties may have affected the chemical characteristics of the vehicles. The zeta potentials of the two types of carrier were approximately $+20 \mathrm{mV}$, and the positive surface charge could have enhanced the combination of the vectors with the negative charged cell surfaces and facilitated endocytosis.

Con A was the first legume lectin recognized as one of the mannose-specific lectins, and is believed to play a role in recognition of mannose-containing vectors. ${ }^{39,40}$ It has been used extensively for evaluation of glycoconjugates. ${ }^{41,42}$ Binding of the terminal $\alpha$-mannose residues to Con $A$ causes agglutination of the complexes in solution, resulting in an increase in turbidity. As shown in Figure 4, when incubated with Con A solution, both A-MN-SLN/DNA and B-MNSLN/DNA appeared to show an increase in turbidity, whereas SLN and SLN/DNA formulations appeared to show no significant increase in turbidity. This could be evidence of the success of mannan-containing ligands modified onto an SLN surface using both premodification and postmodification methods.

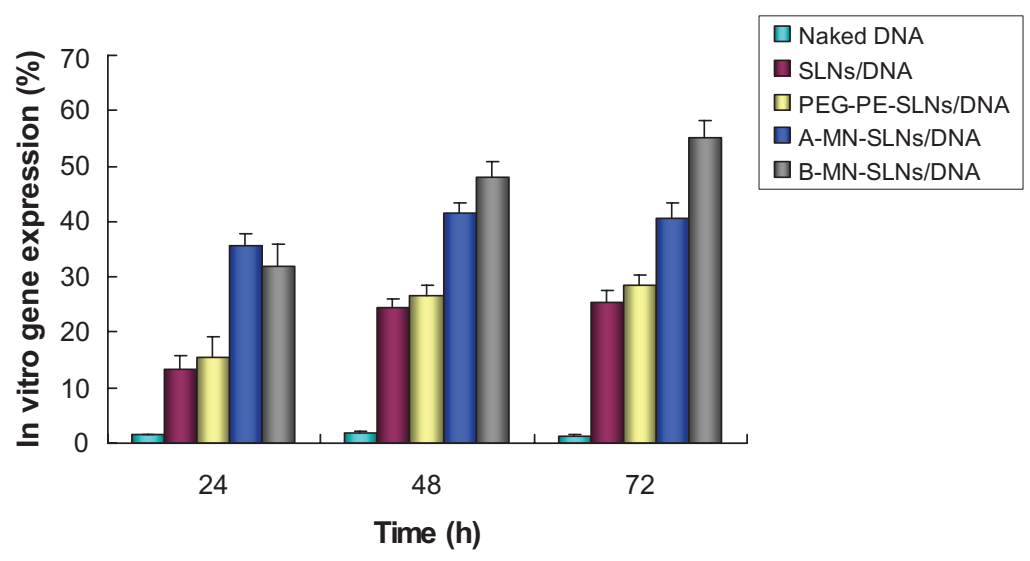

Figure 7 In vitro transfection efficiency of A-MN-SLN/DNA and B-MN-SLN/DNA in Kupffer cells after transfection for 24, 48, and 72 hours. Abbreviations: SLN, solid lipid nanoparticles; A-MN-SLNs/DNA, pre-MN-PEG-PE-modified SLN/DNA; B-MN-SLNs/DNA, post-MN-PEG-PE-modified SLN/DNA; MN, mannan; PEG, polyethylene glycol; PE, L- $\alpha$-phosphatidylethanolamine. 
A
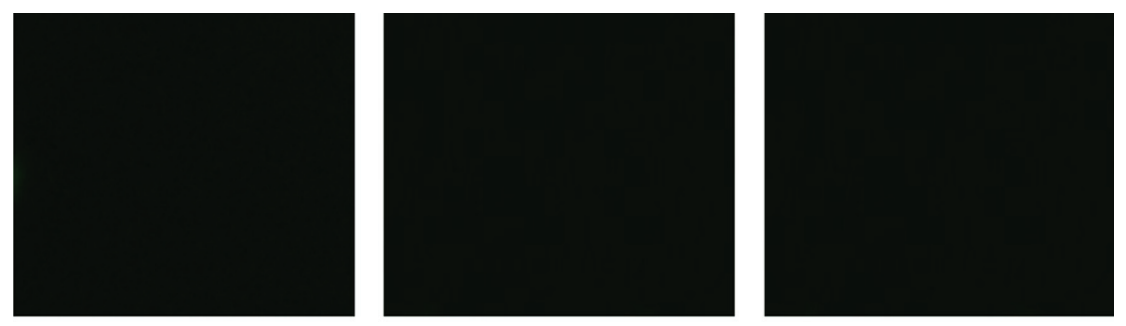

B
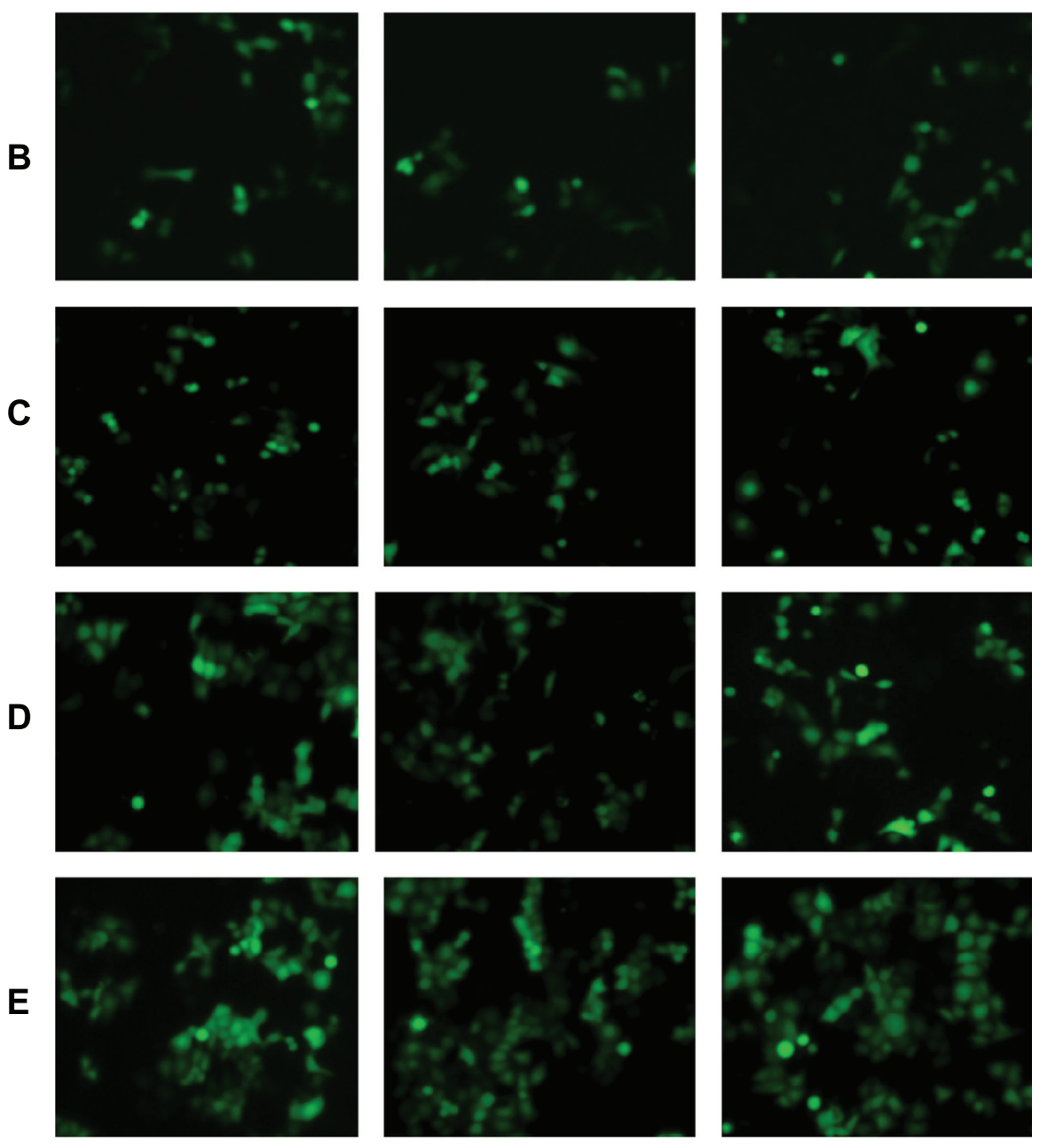

24

48

72

Time (hours)

Figure 8 Fluorescent images of Kupffer cells transfected with naked pEGFP (A), SLN/DNA (B), PEG-PE-SLN/DNA (C), A-MN-SLN/DNA (D), and B-MN-SLN/DNA (E) at 24,48 , and 72 hours after transfection.

Abbreviations: SLN, solid lipid nanoparticles; A-MN-SLNs/DNA, pre-MN-PEG-PE-modified SLN/DNA; B-MN-SLNs/DNA, post-MN-PEG-PE-modified SLN/DNA; MN, mannan; PEG, polyethylene glycol; PE, L- $\alpha$-phosphatidylethanolamine; pEGFP, plasmid-enhanced green fluorescent protein.

An in vitro release study was carried out in order to show the release profiles of the two kinds of vectors. The release of premodified carriers was faster during the 48 hours of study (Figure 6), which may be because the complex was not as tight as for the postmodified vehicles, and DNA release from SLN was easier. After 48 hours, the accumulated gene release from B-MN-SLN/DNA caught up with the steps of A-MN-SLN/DNA.
Both the premodified and postmodified SLN/DNA reached over $80 \%$ gene release at 72 hours. Could the faster release of A-MN-SLN/DNA during 48 hours win some efficiency at the same time points in transfection studies? Or maybe the compact B-MN-SLN/DNA would be more effective?

Kupffer cells are liver-specific resident macrophages that play an integral part in the physiological homeostasis 


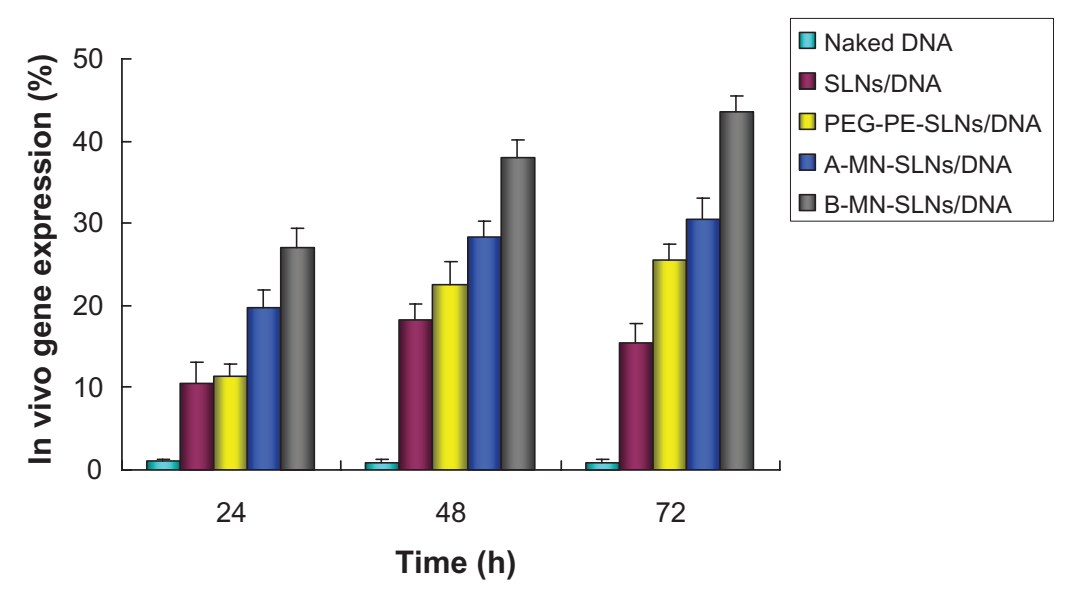

Figure 9 In vivo transfection efficiency of A-MN-SLN/DNA and B-MN-SLN/DNA in Kupffer cells after transfection for 24, 48, and 72 hours. Abbreviations: SLN, solid lipid nanoparticles; A-MN-SLNs/DNA, pre-MN-PEG-PE-modified SLN/DNA; B-MN-SLNs/DNA, post-MN-PEG-PE-modified SLN/DNA; MN, mannan; PEG, polyethylene glycol; PE, L- $\alpha$-phosphatidylethanolamine.

of the liver. In this study, rat Kupffer cells were isolated and used as model cells for in vitro cytotoxicity evaluation and transfection analysis. As shown in Figure 7, modified carriers showed obviously higher efficiency than PEG-PE- SLN/ DNA, unmodified SLN/DNA, or naked DNA $(P<0.05)$. At 24 hours after transfection, the transfection ability of A-MN-SLN/DNA and B-MN-SLN/DNA was almost the same $(P>0.05)$. A-MN-SLN/DNA showed higher transfection efficiency than B-MN-SLN/DNA at 48 and 72 hours following transfection $(P<0.05)$. The results are interesting here because the in vitro release of A-MN-SLN/DNA was less than for B-MN-SLN/DNA at 48 hours, while the in vitro transfection efficiency was better. Could this phenomenon be evidence of better targeting ability for the postmodified system? This question should be answered on completion of our in vivo gene delivery studies.

In vivo gene delivery and expression assays were used in an animal model to compare the gene-delivering ability of premodified and postmodified gene vectors. After intravenous injection, rats in this study were euthanized, and their Kupffer cells were isolated and analyzed using inverted fluorescence microscopy (Figure 8) and flow cytometry (Figure 9). B-MN-SLN/DNA showed better transfection efficiency than A-MN-SLN/DNA at 24, 48, and 72 hours. These results demonstrate that the postmodified SLN had more significant targeting ability for rat liver Kupffer cells, could deliver more genes into the cells, and displayed higher transfection efficiency. The high transfection efficiency at 72 hours after injection indicates the controlled-release features of the vehicles.

This in vitro and in vivo evidence of gene transfection strongly supports the active targeting ability of mannancontaining PEG-PE-modified gene-loaded SLN. Moreover, the results indicate that postmodified gene carriers are superior to premodified gene vectors, although the latter are also promising for targeted gene delivery. This observation will guide our future research, and could be a reference for modification of nanocarriers for targeted gene delivery.

\section{Conclusion}

The results of this study are consistent with the observations of other researchers in this area, ie, that mannose-mediated targeting can successfully deliver genes into cells that express the mannose receptor. We conclude that postmodification of gene-loaded nanomedicine has an advantage over premodification. By carefully formulating the carriers with an optimal ratio of MN-PEG-PE, postmodified vehicles displayed more efficient gene expression in rat Kupffer cells both in vitro and in vivo. This evidence strongly supports the active targeting ability of mannan-containing PEG-PEmodified gene carriers. Most importantly, the proof of superiority of postmodified gene delivery vectors has provided a reference point for further studies on this area.

\section{Disclosure}

The authors report no conflicts of interest in this work.

\section{References}

1. Pathak A, Vyas SP, Gupta KC. Nano-vectors for efficient liver specific gene transfer. Int J Nanomedicine. 2008;3(1):31-49.

2. Torchilin VP. Targeted pharmaceutical nanocarriers for cancer therapy and imaging. AAPS J. 2007;9(2):E128-E147.

3. Kim SK, Park KM, Singha K, et al. Galactosylated cucurbituril-inclusion polyplex for hepatocyte-targeted gene delivery. Chem Commun (Camb). 2010;46(5):692-694.

4. Boulaiz H, Marchal JA, Prados J, Melguizo C, Aránega A. Non-viral and viral vectors for gene therapy. Cell Mol Biol (Noisy-le-grand). 2005;51(1):3-22. 
5. Zhao XB, Lee RJ. Tumor-selective targeted delivery of genes and antisense oligodeoxyribonucleotides via the folate receptor. Adv Drug Deliv Rev. 2004;56(8):1193-1204.

6. Torchilin VP. Multifunctional nanocarriers. Adv Drug Deliv Rev. 2006;58(14):1532-1555.

7. Yu W, Zhang N. Surface modification of nanocarriers for cancer therapy. Curr Nanosci. 2009;5(2):123-134.

8. Li SD, Huang L. Surface-modified LPD nanoparticles for tumor targeting. Ann N Y Acad Sci. 2006;1082:1-8.

9. Juillerat-Jeanneret L, Schmitt F. Chemical modification of therapeutic drugs or drug vector systems to achieve targeted therapy: looking for the grail. Med Res Rev. 2007;27(4):574-590.

10. Chavanpatil MD, Khdair A, Panyam J. Nanoparticles for cellular drug delivery: mechanisms and factors influencing delivery. $J$ Nanosci Nanotechnol. 2006;6(9-10):2651-2663.

11. Peeters L, Sanders NN, Jones A, Demeester J, De Smedt SC. Postpegylated lipoplexes are promising vehicles for gene delivery in RPE cells. J Control Release. 2007;121(3):208-217.

12. Kolhatkar RB, Kitchens KM, Swaan PW, Ghandehari H. Surface acetylation of polyamidoamine (PAMAM) dendrimers decreases cytotoxicity while maintaining membrane permeability. Bioconjug Chem. 2007;18(6):2054-2060.

13. Kojima C, Kono K, Maruyama K, Takagishi T. Synthesis of polyamidoamine dendrimers having poly(ethylene glycol) grafts and their ability to encapsulate anticancer drugs. Bioconjug Chem. 2000;11(6):910-917.

14. Patri AK, Kukowska-Latallo JF, Baker JR Jr. Targeted drug delivery with dendrimers: comparison of the release kinetics of covalently conjugated drug and non-covalent drug inclusion complex. Adv Drug Deliv Rev. 2005;57(15):2203-2214.

15. Forrest ML, Zhao A, Won CY, Malick AW, Kwon GS. Lipophilic prodrugs of Hsp90 inhibitor geldanamycin for nanoencapsulation in poly(ethylene glycol)-b-poly(epsilon-caprolactone) micelles. J Control Release. 2006;116(2):139-149.

16. Weiss B, Schneider M, Muys L, et al. Coupling of biotin-(poly(ethylene glycol))amine to poly(D,L-lactide-co-glycolide) nanoparticles for versatile surface modification. Bioconjug Chem. 2007;18(4):1087-1094.

17. Li SD, Chen YC, Hackett MJ, Huang L. Tumor-targeted delivery of siRNA by self-assembled nanoparticles. Mol Ther. 2008;16(1):163-169.

18. Yamada M, Nishikawa M, Kawakami S, et al. Tissue and intrahepatic distribution and subcellular localization of a mannosylated lipoplex after intravenous administration in mice. J Control Release. 2004;98(1):157-167.

19. Kitani H, Takenouchi T, Sato M, Yoshioka M, Yamanaka N. A novel isolation method for macrophage-like cells from mixed primary cultures of adult rat liver cells. J Immunol Methods. 2010;360(1-2):47-55.

20. Yu W, Liu C, Liu Y, Zhang N, Xu W. Mannan-modified solid lipid nanoparticles for targeted gene delivery to alveolar macrophages. Pharm Res. 2010;27(8):1584-1596.

21. Lobovkina T, Jacobson GB, Gonzalez-Gonzalez E, et al. In vivo sustained release of siRNA from solid lipid nanoparticles. ACS Nano. 2011;5(12):9977-9983.

22. Zhang S, Yun J, Shen S, et al. Formation of solid lipid nanoparticles in a microchannel system with a cross-shaped junction. Chem Eng Sci. 2008;63(23):5600-5605.

23. Ahn SJ, Costa J, Emanuel JR. PicoGreen quantitation of DNA: effective evaluation of samples pre- or post-PCR. Nucleic Acids Res. 1996;24(13):2623-2635.
24. Ren $X, X u$ QH. Label-free DNA sequence detection with enhanced sensitivity and selectivity using cationic conjugated polymers and PicoGreen. Langmuir. 2009;25(1):43-47.

25. Ikeda Y, Iwakiri S, Yoshimori T. Development and characterization of a novel host cell DNA assay using ultra-sensitive fluorescent nucleic acid stain "PicoGreen". J Pharm Biomed Anal. 2009;49(4): 997-1002.

26. Lee JY, Cheong IW, Kim JH. Agglutination study of poly(allyl-alpha-Dglucopyranose/styrene) latex particles in the presence of concanavalin A. Colloids Surf B Biointerfaces. 2005;41(2-3):203-208.

27. Cui Z, Han SJ, Huang L. Coating of mannan on LPD particles containing HPV E7 peptide significantly enhances immunity against HPV-positive tumor. Pharm Res. 2004;21(6):1018-1025.

28. Cui Z, Mumper RJ. Topical immunization using nanoengineered genetic vaccines. J Control Release. 2002;81(1-2):173-184.

29. Kong F, Zhou F, Ge L, Liu X, Wang Y. Mannosylated liposomes for targeted gene delivery. Int J Nanomedicine. 2012;7:1079-1089.

30. Gao Y, Gu W, Chen L, Xu Z, Li Y. A multifunctional nano device as non-viral vector for gene delivery: in vitro characteristics and transfection. J Control Release. 2007;118(3):381-388.

31. Yu W, Liu C, Ye J, Zou W, Zhang N, Xu W. Novel cationic SLN containing a synthesized single-tailed lipid as a modifier for gene delivery. Nanotechnology. 2009;20(1):215102.

32. van Vlerken LE, Vyas TK, Amiji MM. Poly(ethylene glycol)-modified nanocarriers for tumor-targeted and intracellular delivery. Pharm Res. 2007;24(8):1405-1414.

33. Lukyanov AN, Gao Z, Mazzola L, Torchilin VP. Polyethylene glycoldiacyl lipid micelles demonstrate increased accumulation in subcutaneous tumors in mice. Pharm Res. 2002;19(10):1424-1429.

34. Torchilin VP. Recent advances with liposomes as pharmaceutical carriers. Nat Rev Drug Discov. 2005;4(2):145-160.

35. Torchilin VP. Micellar nanocarriers: pharmaceutical perspectives. Pharm Res. 2007;24(1):1-16.

36. Yang L, Wang L, Su XQ, et al. Suppression of ovarian cancer growth via systemic administration with liposome-encapsulated adenovirusencoding endostatin. Cancer Gene Ther. 2010;17(1):49-57.

37. Yu W, Zhang N, Li C. Saccharide modified pharmaceutical nanocarriers for targeted drug and gene delivery. Curr Pharm Des. 2009;15(32): 3826-3836.

38. Sawant RM, Hurley JP, Salmaso S, et al. "SMART" drug delivery systems: double-targeted $\mathrm{pH}$-responsive pharmaceutical nanocarriers. Bioconjug Chem. 2006;17(4):943-949.

39. Summer JB, Howell SF. The identification of the hemagglutinin of the jack bean with concanavalin A. J Bacteriol. 1936;32(2):227-237.

40. Barre A, Bourne Y, Van Damme EJ, Peumans WJ, Rouge P. Mannose-binding plant lectins: different structural scaffolds for a common sugar-recognition process. Biochimie. 2001;83(7):645-651.

41. Kizhakkedathu JN, Creagh AL, Shenoi RA, et al. High molecular weight polyglycerol-based multivalent mannose conjugates. Biomacromolecules. 2010;11(10):2567-2575.

42. Wolfenden ML, Cloninger MJ. Mannose/glucose-functionalized dendrimers to investigate the predictable tunability of multivalent interactions. J Am Chem Soc. 2005;127(35):12168-12169. 


\section{Supplementary data}

\section{In vivo gene delivery (Figure 9)}

Details of the experiments and data analysis are as follows: Cells were washed in cold phosphate-buffered saline three times and the fluorescence intensity of the cells was determined using a flow cytometer equipped with a $488 \mathrm{~nm}$ argon laser for excitation. For each sample, about 10,000 events were collected and fluorescence was detected. Signals were amplified in logarithmic mode for fluorescence to determine the positive events by a standard gating technique. The percentage of positive events was calculated as the events within the gate divided by the total number of events, excluding cell debris. The percentage of cells transfected with plasmid-enhanced green fluorescent protein appeared at the upper right and lower right quadrants of the diagram. The data for the quadrantal diagram of the in vivo study are as follows:

1. Naked plasmid-enhanced green fluorescent protein 24 hours post transfection

Quad X mean Y mean Events\%Total\%Gated

1. UL 6.818 .350 .0474 .01

2. UR $107.678 .6310 .26 \mathbf{1 . 1 8}$

3. LL 2.48 .51198697 .4224 .33

4. LR $43.814 .1650 .54 \mathbf{0 . 4 7}$

Gene expression UR + LR $=1.65 \%$

2. Naked plasmid-enhanced green fluorescent protein 48 hours post transfection

Quad X mean Y mean Events\%Total\%Gated

1. UL 6.818 .350 .0469 .27

2. UR $107.678 .6310 .26 \mathbf{2 . 0 3}$

3. LL 2.48 .51198697 .4228 .38

4. LR $43.814 .1650 .54 \mathbf{0 . 3 2}$

Gene expression: $\mathrm{UR}+\mathrm{LR}=2.35 \%$

3. Naked plasmid-enhanced green fluorescent protein 72 hours post transfection Quad X mean Y mean Events\%Total\%Gated

1. UL 6.818 .350 .0471 .36

2. UR $107.678 .6310 .26 \mathbf{1 . 4 7}$

3. LL 2.48 .51198697 .4226 .39

4. LR $43.814 .1650 .54 \mathbf{0 . 7 5}$

Gene expression: UR + LR $=2.22 \%$

4. SLN/DNA 24 hours post transfection

Quad X mean Y mean Events\%Total\%Gated

1. UL 6.818 .350 .0461 .72

2. UR $107.678 .6310 .26 \mathbf{5 . 5 6}$
3. LL 2.48 .51198697 .4227 .86

4. LR 43.814 .1650 .544 .86

Gene expression: UR + LR $=10.42 \%$

5. SLN/DNA 48 hours post transfection Quad X mean Y mean Events\%Total\%Gated

1. UL 6.818 .350 .0458 .24

2. UR $107.678 .6310 .26 \mathbf{1 2 . 4 1}$

3. LL 2.48 .51198697 .4223 .79

4. LR $43.814 .1650 .54 \mathbf{5 . 5 2}$

Gene expression: UR $+\mathrm{LR}=17.93 \%$

6. SLN/DNA 72 hours post transfection

Quad X mean Y mean Events\%Total\%Gated

1. UL 6.818 .350 .0458 .24

2. UR $107.678 .6310 .26 \mathbf{1 2 . 4 1}$

3. LL 2.48 .51198697 .4223 .79

4. LR $43.814 .1650 .54 \mathbf{5 . 5 2}$

Gene expression: UR + LR $=17.93 \%$

7. PEG-PE-SLN/DNA 24 hours post transfection Quad X mean Y mean Events\%Total\%Gated

1. UL 6.818 .350 .0470 .06

2. UR 107.6 $78.6310 .26 \mathbf{6 . 4 0}$

3. LL 2.48 .51198697 .4218 .61

4. LR 43.814 .1650 .544 .93

Gene expression: UR $+\mathrm{LR}=11.33 \%$

8. PEG-PE-SLN/DNA 48 hours post transfection Quad X mean Y mean Events\%Total\%Gated

1. UL 6.818 .350 .0452 .16

2. UR $107.678 .6310 .26 \mathbf{1 6 . 8 7}$

3. LL 2.48 .51198697 .4225 .30

4. LR $43.814 .1650 .54 \mathbf{5 . 6 5}$

Gene expression: UR + LR $=22.52 \%$

9. PEG-PE-SLN/DNA 72 hours post transfection Quad X mean Y mean Events\%Total\%Gated

1. UL 6.818 .350 .0449 .35

2. UR $107.678 .6310 .26 \mathbf{1 9 . 0 8}$

3. LL 2.48 .51198697 .4225 .05

4. LR $43.814 .1650 .54 \mathbf{6 . 5 2}$

Gene expression: UR + LR $=25.6 \%$

10. A-MN-SLN/DNA 24 hours post transfection Quad X mean Y mean Events\%Total\%Gated

1. UL 6.818 .350 .0457 .53

2. UR $107.678 .6310 .26 \mathbf{1 1 . 4 2}$

3. LL 2.48 .51198697 .4222 .82

4. LR $43.814 .1650 .54 \mathbf{8 . 2 3}$

Gene expression: UR $+\mathrm{LR}=19.65 \%$ 
11. A-MN-SLN/DNA 48 hours post transfection Quad X mean Y mean Events\%Total\%Gated

1. UL 6.818 .350 .0446 .36

2. UR $107.678 .6310 .26 \mathbf{2 2 . 4 8}$

3. LL 2.48 .51198697 .4225 .25

4. LR $43.814 .1650 .54 \mathbf{5 . 8 8}$

Gene expression: UR + LR $=28.36 \%$

12. A-MN-SLN/DNA 72 hours post transfection Quad X mean Y mean Events\%Total\%Gated

1. UL 6.818 .350 .0448 .78

2. UR $107.678 .6310 .26 \mathbf{2 0 . 9 3}$

3. LL 2.48 .51198697 .4220 .77

4. LR $43.814 .1650 .54 \mathbf{9 . 5 2}$

Gene expression: UR + LR $=30.45 \%$

13. B-MN-SLN/DNA 24 hours post transfection Quad X mean Y mean Events\%Total\%Gated

1. UL 6.818 .350 .0449 .74

2. UR $107.678 .6310 .26 \mathbf{1 7 . 7 8}$

3. LL 2.48 .51198697 .4223 .14

4. LR 43.814 .1650 .549 .34

Gene expression: UR + LR $=27.12 \%$

14. B-MN-SLN/DNA 48 hours post transfection Quad X mean Y mean Events\%Total\%Gated

1. UL 6.818 .350 .0444 .73

2. UR $107.678 .6310 .26 \mathbf{2 5 . 1 5}$

3. LL 2.48 .51198697 .4217 .21

4. LR $43.814 .1650 .54 \mathbf{1 2 . 8 8}$

Gene expression: UR + LR $=38.03 \%$
15. B-MN-SLN/DNA 72 hours post transfection Quad X mean Y mean Events\%Total\%Gated

1. UL 6.818 .350 .0437 .26

2. UR $107.678 .6310 .26 \mathbf{3 2 . 6 7}$

3. LL 2.48 .51198697 .4219 .23

4. LR $43.814 .1650 .54 \mathbf{1 0 . 8 3}$

Gene expression: UR + LR $=43.5 \%$

\section{Abbreviations}

UL, upper left; UR, upper right; LL, lower left; LR, lower right; SLN, solid lipid nanoparticles; A-MN-SLN/DNA, pre-MN-PEG-PE-modified SLN/DNA; B-MN-SLN/DNA, post-MN-PEG-PE-modified SLN/DNA; MN, mannan; PEG, polyethylene glycol; PE, L- $\alpha$-phosphatidylethanolamine; pEGFP, plasmid-enhanced green fluorescent protein.
International Journal of Nanomedicine

\section{Publish your work in this journal}

The International Journal of Nanomedicine is an international, peerreviewed journal focusing on the application of nanotechnology in diagnostics, therapeutics, and drug delivery systems throughout the biomedical field. This journal is indexed on PubMed Central,

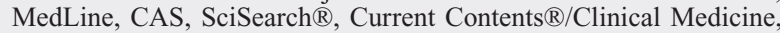

\section{Dovepress}

Journal Citation Reports/Science Edition, EMBase, Scopus and the Elsevier Bibliographic databases. The manuscript management system is completely online and includes a very quick and fair peer-review system, which is all easy to use. Visit http://www.dovepress.com/ testimonials.php to read real quotes from published authors. 